Supporting Information

\title{
Uncommon Pyrazoyl-Carboxyl Bifunctional Ligand-Based \\ Microporous Lanthanide Systems: Sorption, and Luminescent Sensing Properties
}

\author{
Gao-Peng Li, ${ }^{\dagger}$ Ge Liu, ${ }^{\dagger}$ Yong-Zhi Li, ${ }^{\dagger}$ Lei Hou, ${ }^{\dagger, *}$ Yao-Yu Wang, ${ }^{\dagger}$ and Zhonghua Zhu ${ }^{\ddagger}$ \\ ${ }^{\dagger}$ Key Laboratory of Synthetic and Natural Functional Molecule Chemistry of the Ministry of Education, Shaanxi \\ Key Laboratory of Physico-Inorganic Chemistry, College of Chemistry \& Materials Science, Northwest University, \\ Xi'an 710069, P. R. China, Email: lhou2009@nwu.edu.cn \\ ${ }^{\ddagger}$ School of Chemical Engineering, The University of Queensland, Brisbane 4072, Australia
}



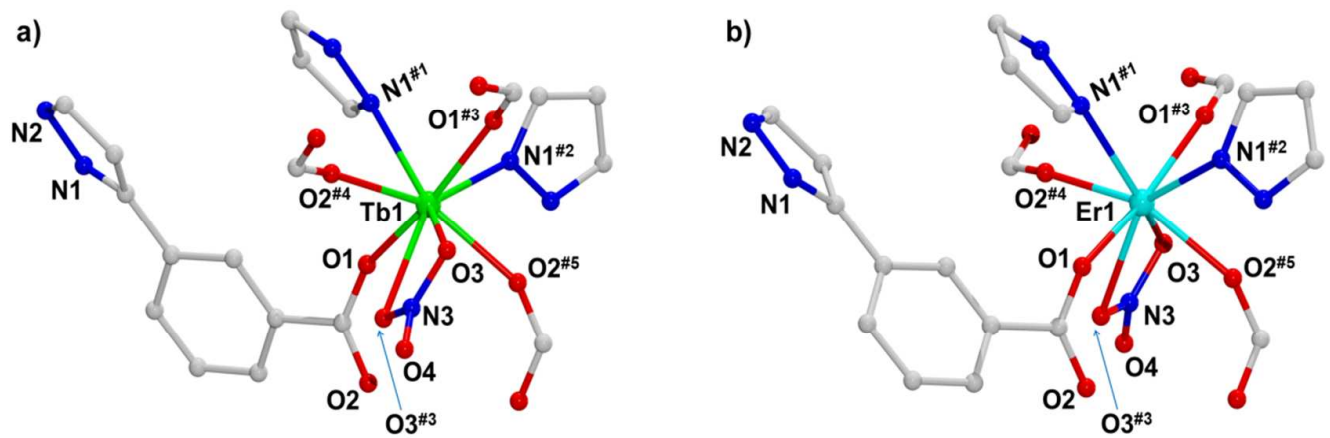

Figure S1. a) Coordination environment of $\mathrm{Tb}^{3+}$ ion in 1-Tb, symmetry codes: $\# 1=0.5-\mathrm{x}, 0.5-\mathrm{y},-\mathrm{z}$; $\# 2=0.5+x, 0.5-y, 0.5+z ; \# 3=1-x, y, 0.5-z ; \# 4=x, 1-y, z-0.5 ; \# 5=1-x, 1-y, 1-z$. b) Coordination environment of $\mathrm{Er}^{3+}$ ion in 1-Er, symmetry codes: $\# 1=0.5-\mathrm{x}, 0.5-\mathrm{y}, 2-\mathrm{z} ; \# 2=\mathrm{x}-0.5,0.5-\mathrm{y}, \mathrm{z}-0.5$; \#3 $=-\mathrm{x}, \mathrm{y}, 1.5-\mathrm{z} ; \# 4=\mathrm{x},-\mathrm{y}, \mathrm{z}+0.5 ; \# 5=-\mathrm{x},-\mathrm{y}, 1-\mathrm{z}$.
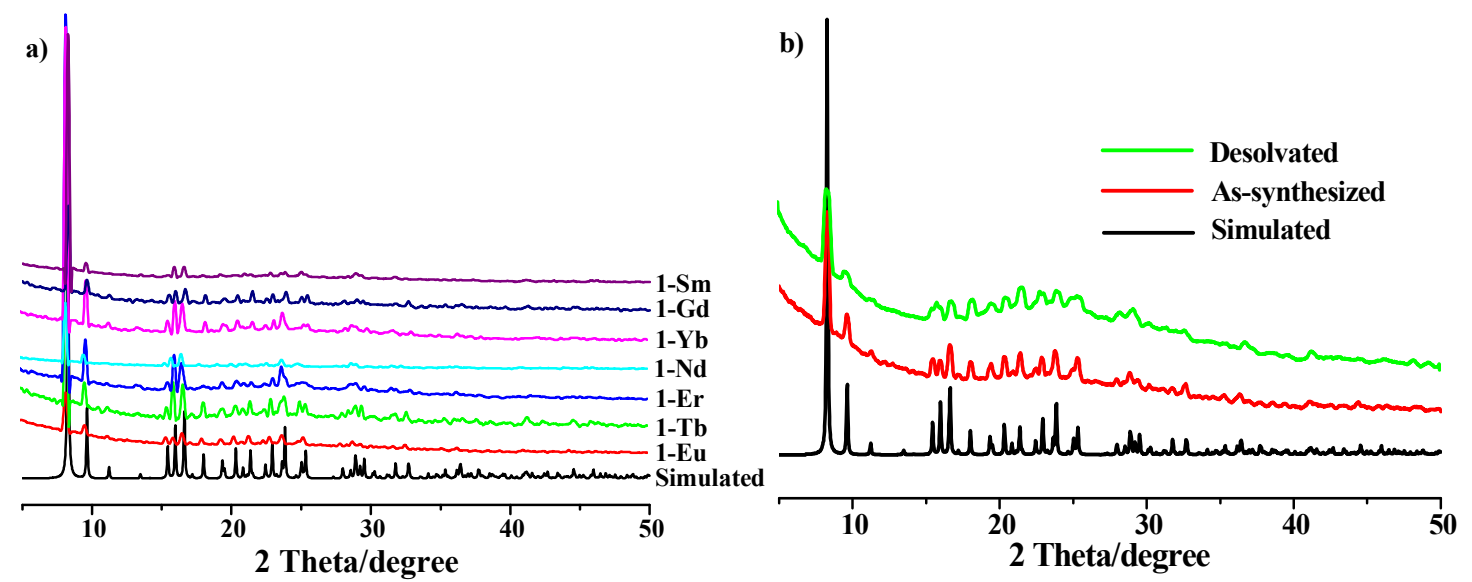

Figure S2. a) PXRD patterns of 1-Eu simulated from the X-ray single-crystal structure and assynthesized samples of 1-Eu, 1-Tb, 1-Er, 1-Nd, 1-Yb, 1-Smand 1-Gd. The very similar PXRD patters confirmed the isostructural frameworks of eight complexes. b) PXRD patterns of 1-Eu simulated from the X-ray single-crystal structure, as-synthesized and desolvated samples.

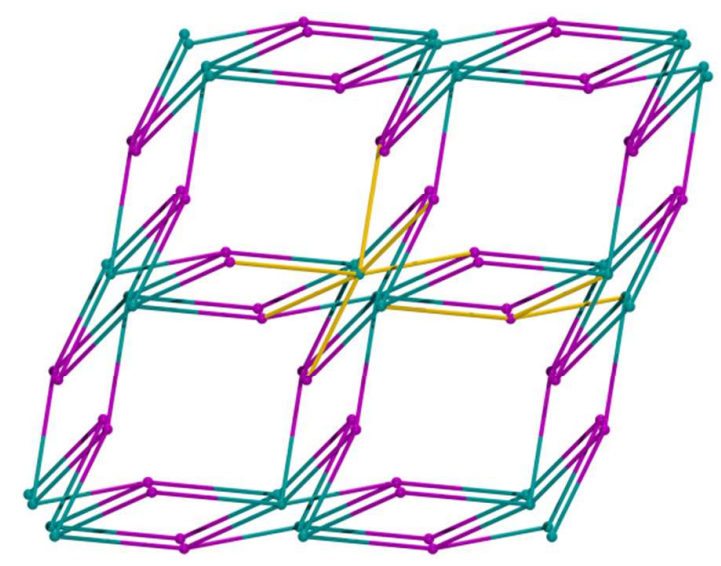

Figure S3. (3,6)-Connected ant topology in 1-Eu. 


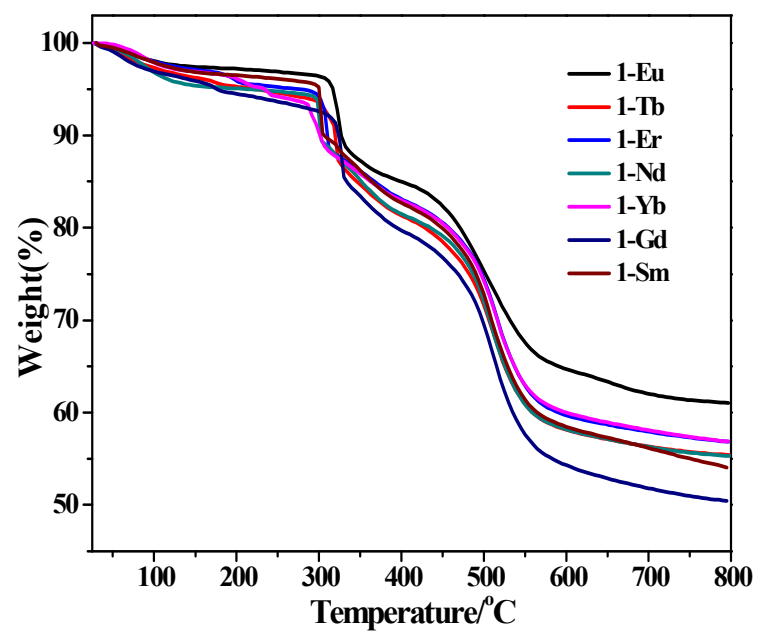

Figure S4. TGA curves of 1-Ln, which showed the similar three-step weight losses under $\mathrm{N}_{2}$ environment, agreeing with their similar structures.
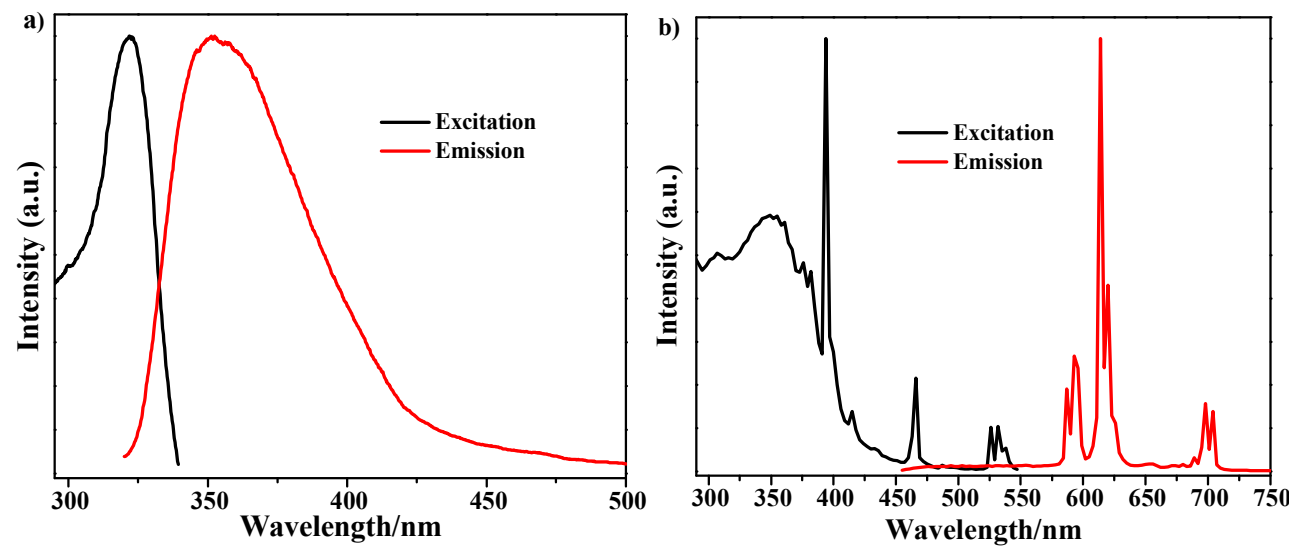

Figure S5. Excitation and emission spectra of $\mathrm{H}_{2} \mathrm{pzbc}$ (a) and 1-Eu (b).

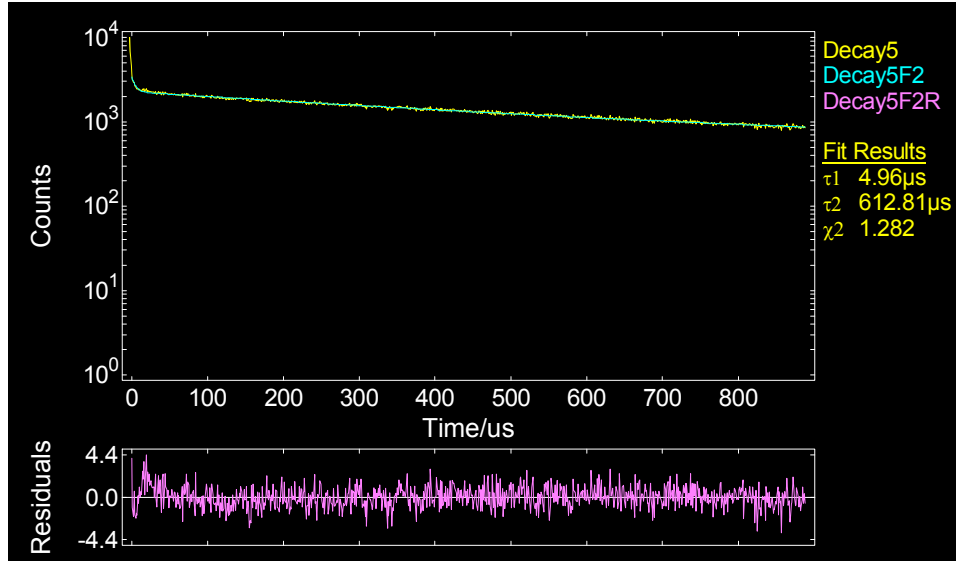

Figure S6. Luminescence decay of 1-Eu measured at the excitation/emission maxima, which can be fitted with two-exponential decay: $\tau_{1}=4.96 \mu \mathrm{s}(0.61 \%), \tau_{1}=612.81 \mu \mathrm{s}(99.39 \%), \chi^{2}=1.282$. 

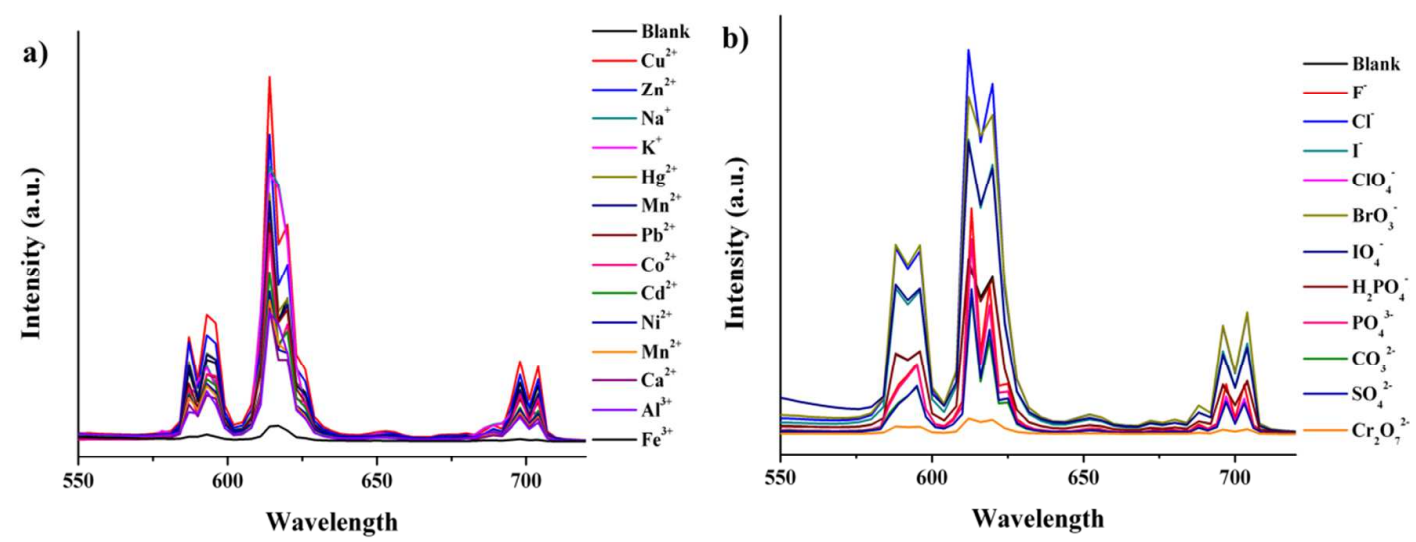

Figure S7. Luminescence spectra of $\mathbf{1 - E \mathbf { u }}$ in $1 \times 10^{-3} \mathrm{M}$ different cation (a) and anion (b) solutions.
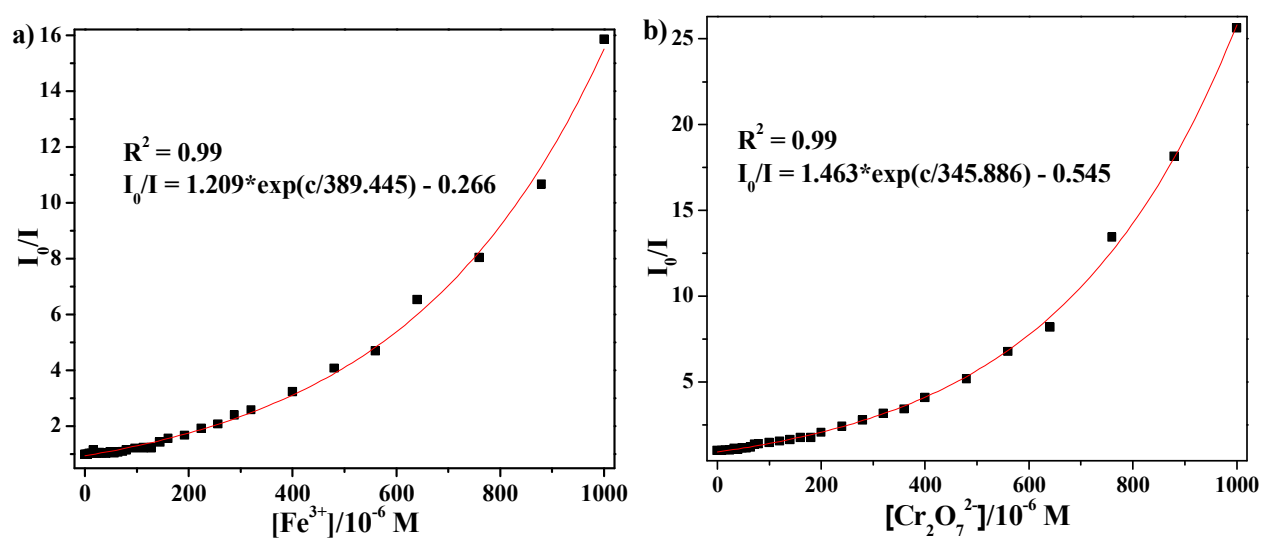

Figure S8. Luminescent intensity $v s\left[\mathrm{Fe}^{3+}\right]$ plot (a), and luminescent intensity $v s\left[\mathrm{Cr}_{2} \mathrm{O}_{7}^{2-}\right]$ plot (b). $\left(\mathrm{I}_{0}\right.$ and $\mathrm{I}$ are the luminescent intensities of 1-Eu in the absence and presence of $\mathrm{Fe}^{3+}$ or $\mathrm{Cr}_{2} \mathrm{O}_{7}{ }^{2-}$, respectively.)
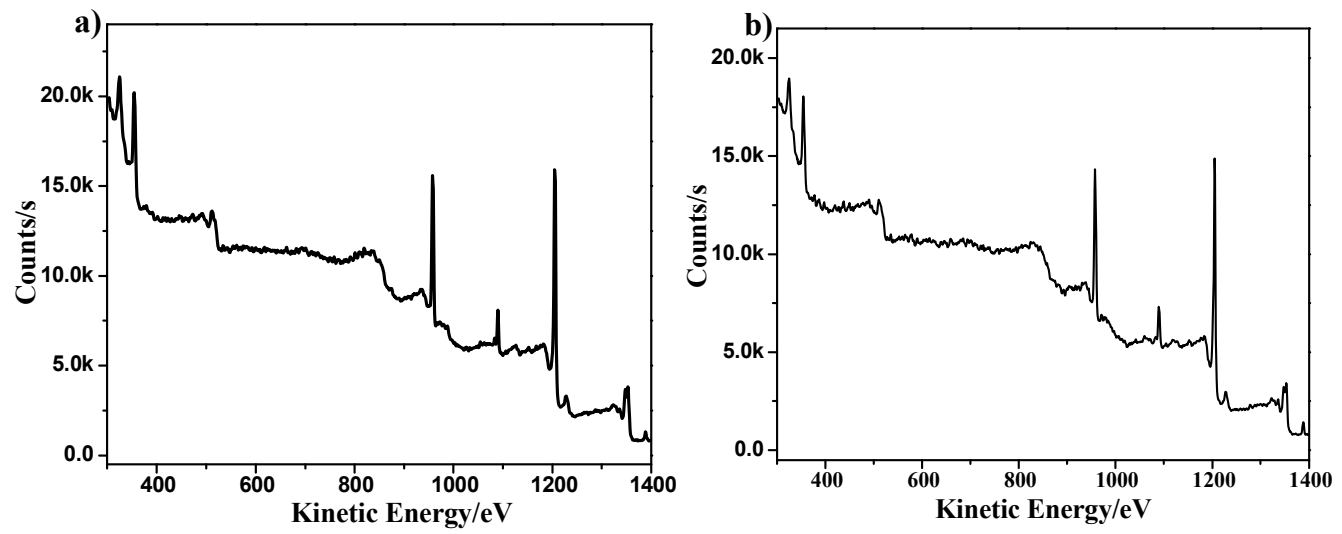

Figure S9. (a) XPS of the recycled 1-Eu in $\mathrm{Fe}^{3+}$ solution (The binding energy of $\mathrm{Fe}^{3+}$ should peak in $707.0 \mathrm{eV}$.). (b) XPS of the recycled 1-Eu in $\mathrm{Cr}_{2} \mathrm{O}_{7}{ }^{2-}$ solution (The binding energy of $\mathrm{Cr}_{2} \mathrm{O}_{7}{ }^{2-}$ should peak in $579.0 \mathrm{eV}$.). 


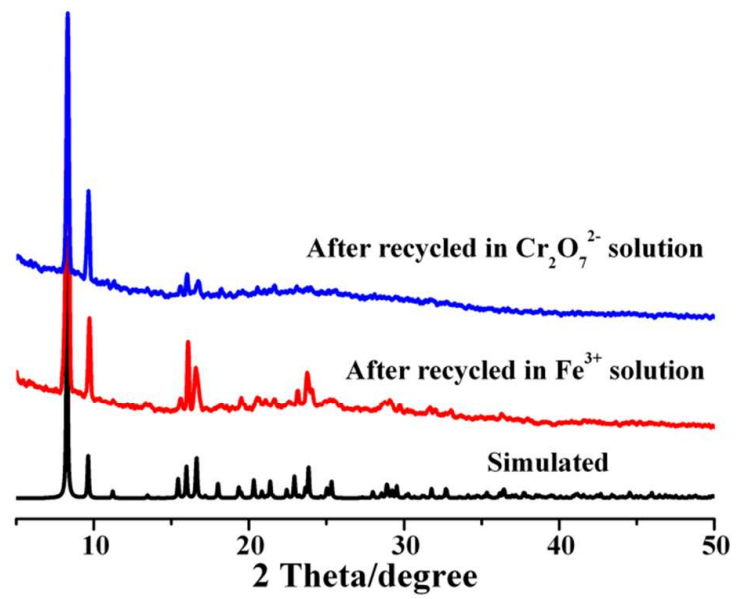

Figure S10. PXRD patterns of 1-Eu after three recycles in $\mathrm{Fe}^{3+}$ and $\mathrm{Cr}_{2} \mathrm{O}_{7}^{2-}$ solutions.
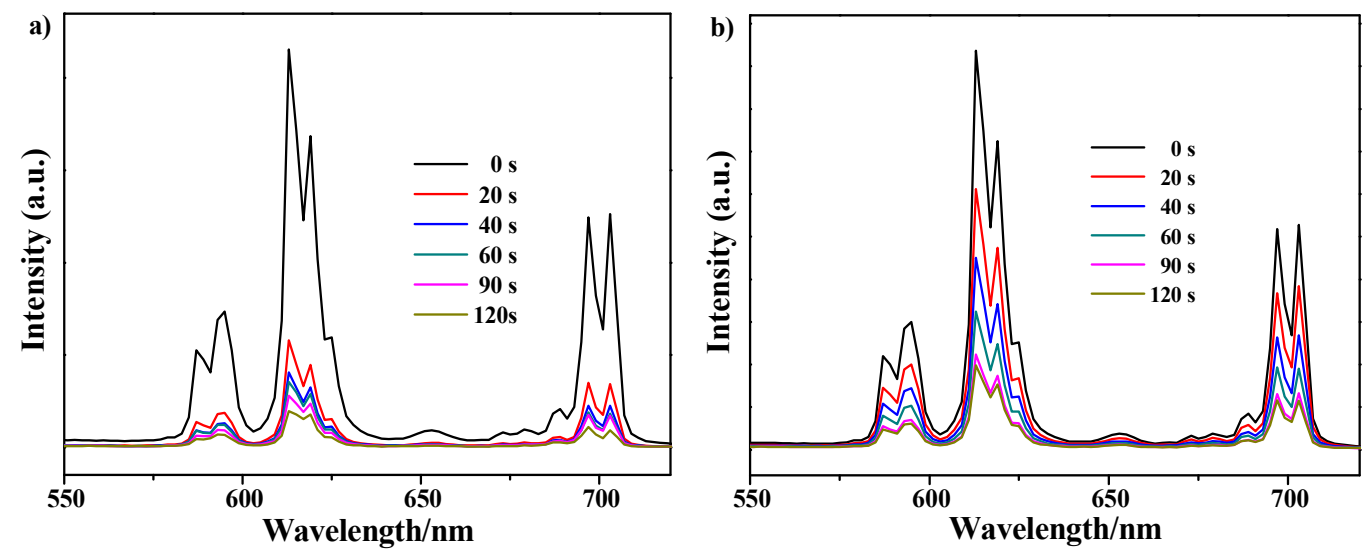

Figure S11. Emission spectra of 1-Eu at different reaction times in $\mathrm{Fe}^{3+}$ (a) and $\mathrm{Cr}_{2} \mathrm{O}_{7}{ }^{2-}$ (b) ethanol solutions.

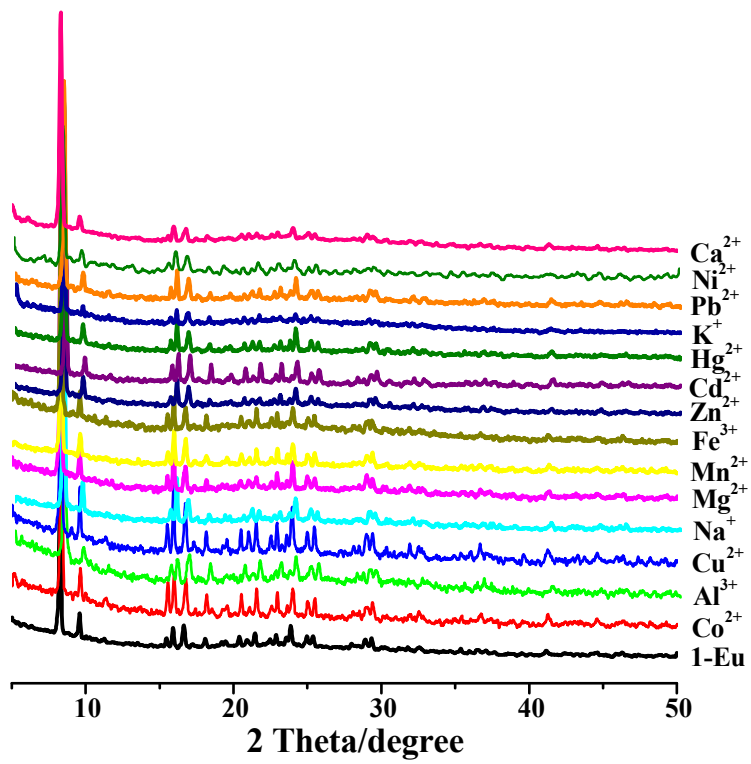

Figure S12. PXRD patterns of 1-Eu treated by different $\mathrm{M}\left(\mathrm{NO}_{3}\right)_{\mathrm{x}}$ ethanol solutions. It indicated that 1-Eu retains its framework after immersed in ethonal solution containing different metal ions. 


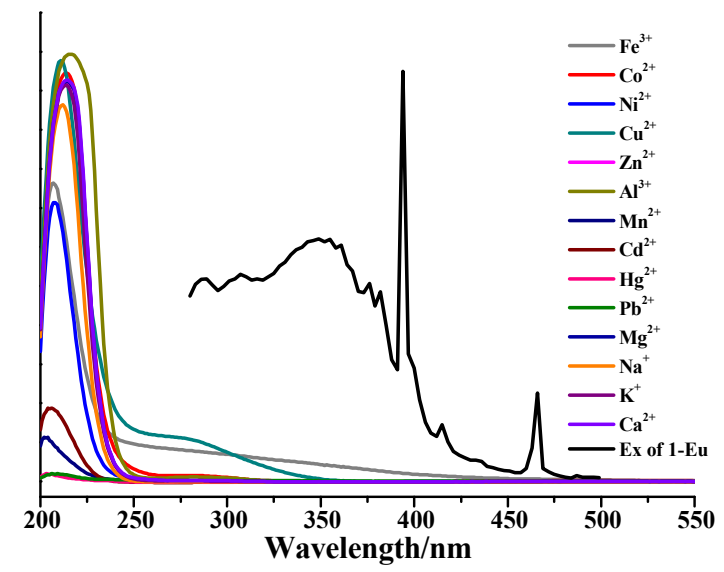

Figure S13. UV-Vis adsorption spectra of $\mathrm{M}\left(\mathrm{NO}_{3}\right)_{\mathrm{X}}$ ethanol solutions and the excitation spectrum of 1-Eu.

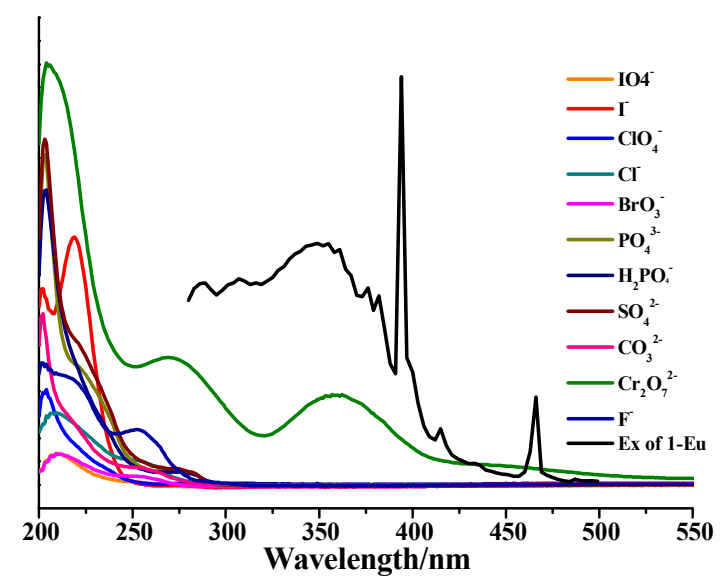

Figure S14. UV-Vis adsorption spectra of $\mathrm{K}(\text { anion })_{\mathrm{x}}$ ethanol solutions and the excitation spectrum of 1-Eu.

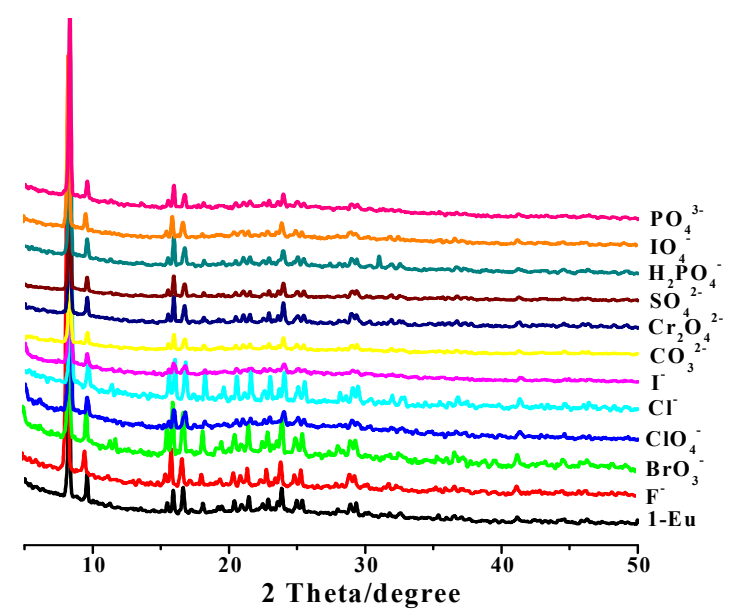

Figure S15. PXRD patterns of 1-Eu treated by different $\mathrm{K}(\text { anion })_{\mathrm{x}}$ ethanol solutions. It indicated that 1-Eu retains its framework after immersed in ethonal solution containing different anions. 


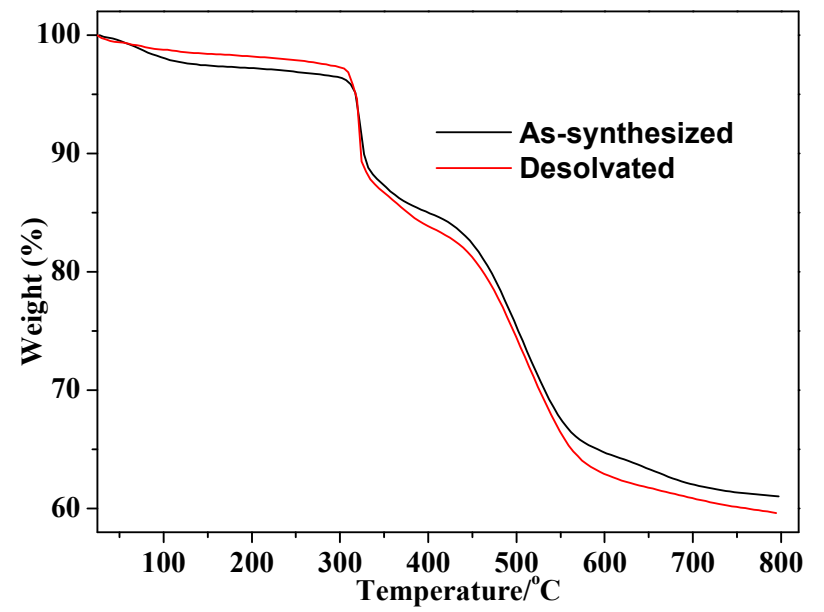

Figure S16. TGA plots of the as-synthesized and desolvated samples of 1-Eu.

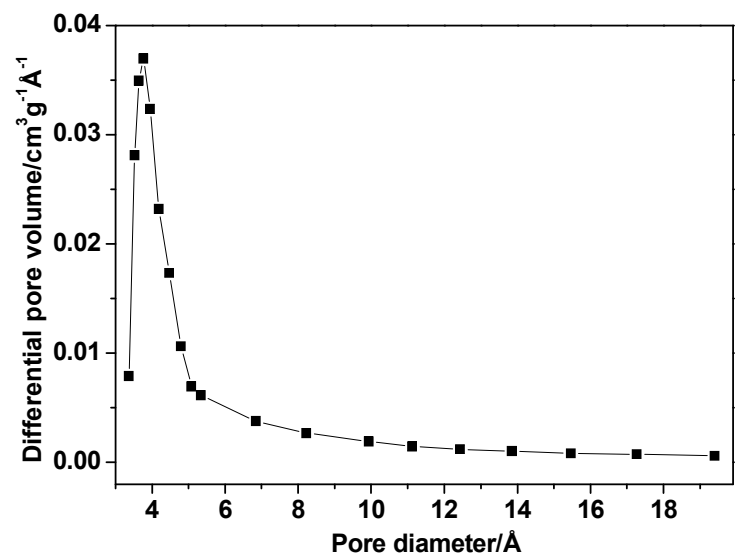

Figure S17. Differential pore volume as a function of pore width calculated from the adsorption isotherm of $\mathrm{CO}_{2}$ at $195 \mathrm{~K}$ using the Horvath-Kawazoe model, the obtained median pore width of $3.9 \AA$ is consistent with pore sizes calculated by single crystal structure.

\section{$\mathrm{CO}_{2} / \mathrm{CH}_{4}$ Selectivity Prediction via IAST}

The experimental isotherm data for pure $\mathrm{CO}_{2}$, and $\mathrm{CH}_{4}$ were fitted using a dual Langmuir-Freundlich (L-F) model:

$$
q=\frac{a_{1}{ }^{*} b_{1} P^{1 / c 1}}{1+b_{1} P^{1 / c 1}}+\frac{a_{2}{ }^{*} b_{2} * P^{1 / c 2}}{1+b_{1} * P^{1 / c 1}}
$$

Where $q$ and $p$ are adsorbed amounts and the pressure of component i, respectively.

The adsorption selectivities for binary mixtures of $\mathrm{CO}_{2} / \mathrm{CH}_{4}$, defined by

$$
S_{\mathrm{i} / \mathrm{j}}=\frac{x_{\mathrm{i}}^{*} y_{\mathrm{j}}}{x_{\mathrm{j}}^{*} y_{\mathrm{i}}}
$$

were respectively calculated using the Ideal Adsorption Solution Theory (IAST) of Myers and Prausnitz. ${ }^{3}$ Where $x_{\mathrm{i}}$ is the mole fraction of component $\mathrm{i}$ in the adsorbed phase and $y_{\mathrm{i}}$ is the mole fraction of component $\mathrm{i}$ in the bulk. 

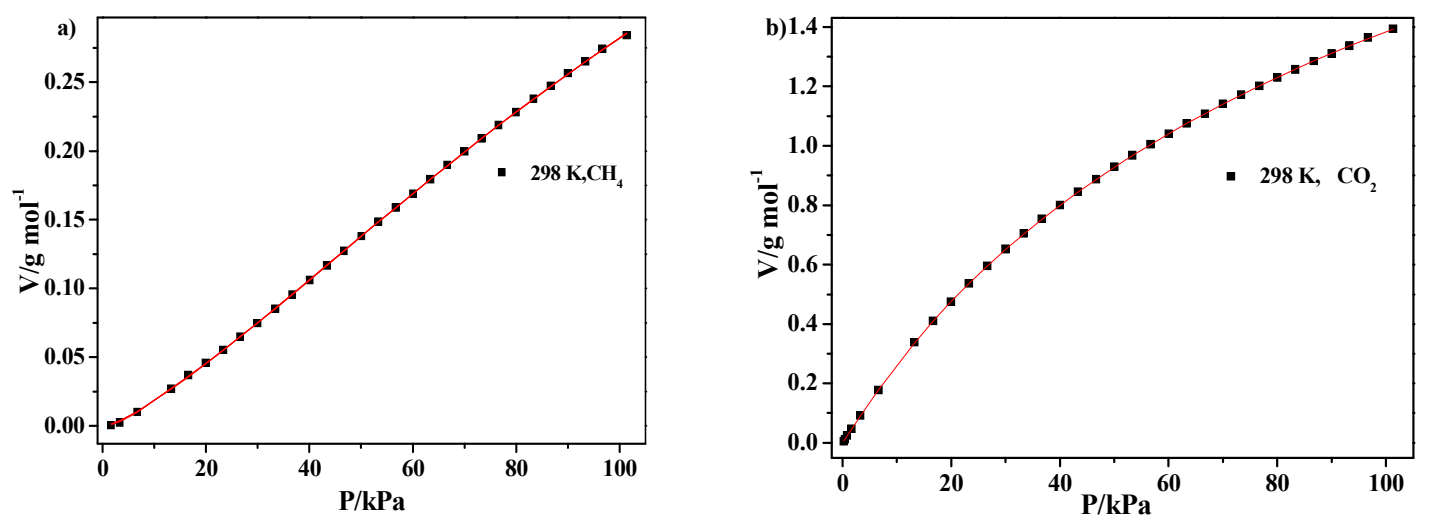

Figure S18. a) $\mathrm{CO}_{2}$ adsorption isotherms of 1-Eu with fitted by dual L-F model: $298 \mathbf{K}$, a1 = $2.85176, \mathrm{~b} 1=0.00968, \mathrm{c} 1=0.97704, \mathrm{a} 2=0.0577, \mathrm{~b} 2=0.00913, \mathrm{c} 2=1.82031, \mathrm{Chi}^{\wedge} 2=9.6793 \mathrm{E}-7$, $\mathrm{R}^{\wedge} 2=1$; b) $\mathrm{CH}_{4}$ adsorption isotherms of $1 \mathrm{a}$ with fitting by L-F model: $\mathbf{2 9 8 K}$, a $1=0.70811$, b1 = $0.00066, \mathrm{c} 1=1.49025, \mathrm{a} 2=0.00753, \mathrm{~b} 2=0.00033, \mathrm{c} 2=3.56723, \mathrm{Chi}^{\wedge} 2=2.0318 \mathrm{E}-7, \mathrm{R}^{\wedge} 2=$ 0.99998 .

\section{Calculation of Sorption Heat for $\mathrm{CO}_{2}$ Uptake Using Virial 2 Model}

$\ln P=\ln N+1 / T \sum_{i=0}^{m} a_{i} N^{i}+\sum_{i=0}^{n} b_{i} N^{i} Q_{\mathrm{st}}=-R \sum_{i=0}^{m} a_{i} N^{i}$

The above virial expression was used to fit the combined isotherm data for 1-Euat 273.15, 285 and $298 \mathrm{~K}$, where $P$ is the pressure, $N$ is the adsorbed amount, $T$ is the temperature, $a_{i}$ and $b_{i}$ are virial coefficients, and $m$ and $N$ are the number of coefficients used to describe the isotherms. Qst is the coverage-dependent enthalpy of adsorption and $\mathrm{R}$ isthe universal gas constant.
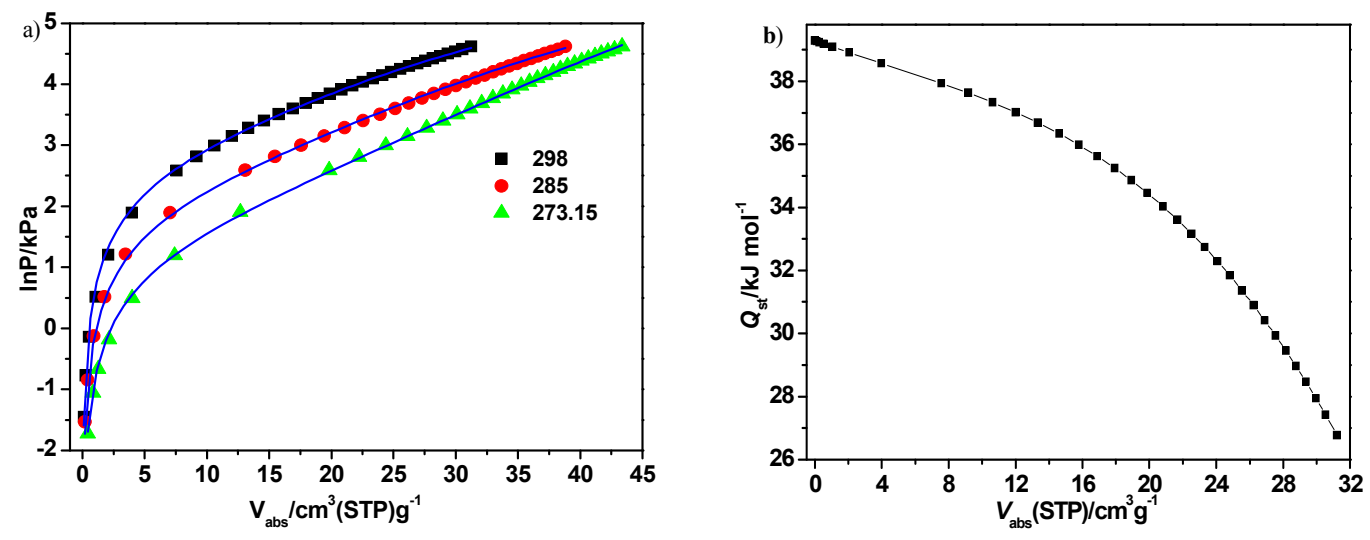

Figure S19. a) $\mathrm{CO}_{2}$ adsorption isotherms for 1-Eu with fitted by Virial 2 model. Fitting results: a0 $=-4725.44169, \mathrm{a} 1=23.30611, \mathrm{a} 2=-0.57602$, $\mathrm{a} 3=0.04384, \mathrm{~b} 0=16.47888, \mathrm{~b} 1=-0.1389, \mathrm{~b} 2=$ $0.00399, \mathrm{~b} 3=-0.00018, \mathrm{Chi}^{\wedge} 2=0.00218, \mathrm{R}^{\wedge} 2=0.99926$. b) $\mathrm{CO}_{2}$ adsorption heat calculated according to the virial equation. 


\section{GCMC Simulation Methodlody}

Grand canonical Monte Carlo (GCMC) simulations were performed for the adsorption of $\mathrm{CO}_{2}$ in 1 aby the Sorption module of Material Studio. ${ }^{1}$ The framework and $\mathrm{CO}_{2}$ molecule were considered to be rigid. The partial charges for carbon and oxygen atoms of $\mathrm{CO}_{2}$ molecules were $0.576 e$ and -0.288 e, respectively. ${ }^{2}$ The partial charges for atoms of 1-Euwere derived from QEq method and QEq neutral1.0 parameter (Table S5). One unit cell was used during the simulations. The interaction energies between $\mathrm{CO}_{2}$ and framework were computed through the Coulomb and Lennard-Jones 6-12 (LJ) potentials. All parameters for $\mathrm{CO}_{2}$ molecule and atoms of 1-Euwere modeled with the universal forcefield (UFF) embedded in the MS modeling package. A cutoff distance of $12.5 \AA$ was used for LJ interactions, and the Coulombic interactions were calculated by using Ewald summation. For each run, the $5 \times 10^{6}$ maximum loading steps, $1 \times 10^{7}$ production steps, automated temperature control in the annealing cycles and 40 temperature cycles were employed.

\section{References}

(1) Accelrys, Materials Studio Getting Started, release 5.0; Accelrys Software, Inc.: San Diego, CA, 2009.

(2) Hirotani, A.; Mizukami, K.; Miura, R.; Takaba, H.; Miya, T.; Fahmi, A.; Stirling, A.; Kuboand M.; Miyamoto, A. Appl. Surf. Sci. 1997, 120, 81-84.

a)

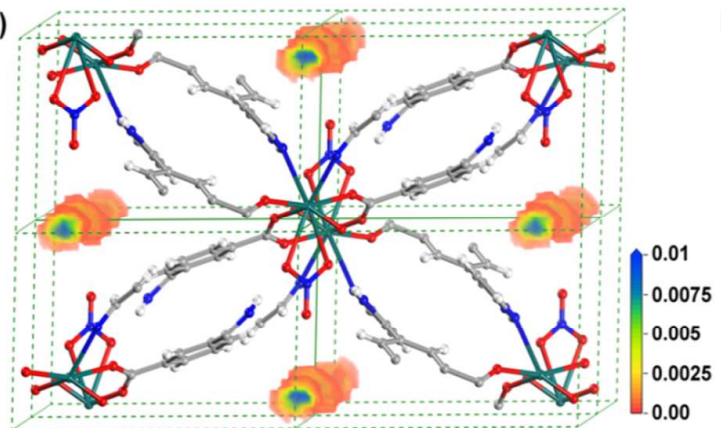

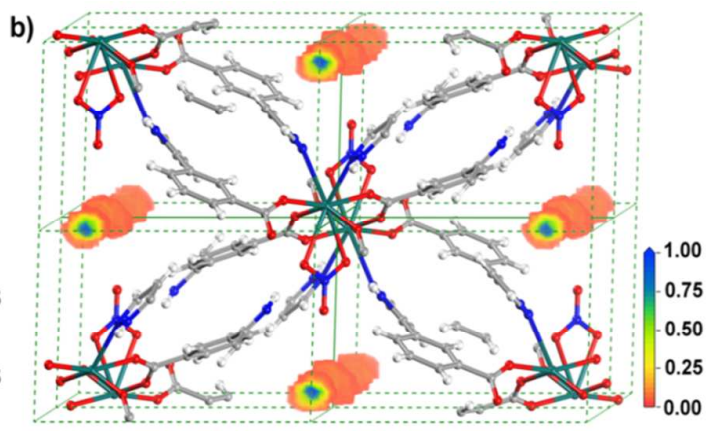

Figure S20. Density contours of $\mathrm{CO}_{2}$ adsorption in the pores of 1-Euobtainedfrom GCMC simulations at $298 \mathrm{~K}$ under pressures 0.1 (a) and $100 \mathrm{kPa}$ (b), respectively. 
Table S1. Selected luminescent Ln-MOFs materials for sensing functionality.

\begin{tabular}{|c|c|c|c|}
\hline Molecular & luminescent substrates & active sites & Ref \\
\hline $\mathrm{Eu}^{3+} @ \mathrm{MIL}-124$ & $\begin{array}{l}\mathrm{Fe}^{2+}, \mathrm{Fe}^{3+}, \mathrm{Cr}_{2} \mathrm{O}_{7}^{2-} \\
\text { organic molecule }\end{array}$ & carboxylate groups & 1 \\
\hline$\left[\mathrm{Eu}(\mathrm{BTPCA})\left(\mathrm{H}_{2} \mathrm{O}\right)\right] \cdot 2 \mathrm{DMF} \cdot 3 \mathrm{H}_{2} \mathrm{O}$ & $\mathrm{Fe}^{3+}$ & triazinyl $\mathrm{N}$ atoms & 2 \\
\hline$\left.\left[\mathrm{Ln}_{2} \mathrm{~L}_{1}\right)_{2}\right] \cdot\left(\mathrm{H}_{2} \mathrm{O}\right)_{3} \cdot\left(\mathrm{Me}_{2} \mathrm{NH}_{2}\right)_{\mathrm{n}}$ & $\mathrm{Fe}^{3+}, \mathrm{Cu}^{2+}$ & carboxylate groups & 3 \\
\hline$\left[\mathrm{Tb}_{2}(\mathrm{BDC})_{3}\left(\mathrm{H}_{3} \mathrm{O}\right)_{4}\right]_{\mathrm{n}}$ & $\mathrm{Cu}^{2+}$ & $* *$ & 4 \\
\hline$\left[\mathrm{H}_{2} \mathrm{~N}\left(\mathrm{CH}_{3}\right)_{2}\right]\left[\mathrm{Eu}\left(\mathrm{H}_{2} \mathrm{O}\right)_{2}(\mathrm{BTMIPA})\right] \cdot 2 \mathrm{H}_{2} \mathrm{O}$ & $\mathrm{Fe}^{3+}, \mathrm{Al}^{3+}$ & {$\left[\mathrm{H}_{2} \mathrm{~N}\left(\mathrm{CH}_{3}\right)_{2}\right]^{+}$} & 5 \\
\hline Tb-DSOA & $\mathrm{Fe}^{3+}$ & culfonateoxygen atoms & 6 \\
\hline$\left[\mathrm{Tb}(\mathrm{Hbtca})\left(\mathrm{H}_{2} \mathrm{O}\right)_{2}\right] \cdot \mathrm{H}_{2} \mathrm{O}$ & $\mathrm{Fe}^{3+}$ & carboxyl groups & 7 \\
\hline$\left[\mathrm{Eu}_{3}(\mathrm{bpydb})_{3}(\mathrm{HCOO})\left(\mu_{3}-\mathrm{OH}\right)_{2}(\mathrm{DMF})\right] \cdot(\mathrm{DMF})_{3} \cdot\left(\mathrm{H}_{2} \mathrm{O}\right)_{2}$ & $\mathrm{Cu}^{2+}$, organic molecule & pyridyl sites & 8 \\
\hline$\left[\operatorname{TbZn}\left(\mathrm{L}_{2}\right)\left(\mathrm{CO}_{3}\right)_{2}\left(\mathrm{H}_{2} \mathrm{O}\right)\right]_{\mathrm{n}}$ & organic molecule & $* *$ & 9 \\
\hline$\left[\mathrm{Tb}_{3}\left(\mathrm{NO}_{3}\right)(\mathrm{BPTA})_{2}\left(\mathrm{H}_{2} \mathrm{O}\right)_{6}\right] \cdot 3 \operatorname{Diox} \cdot 8 \mathrm{H}_{2} \mathrm{O}$ & $\begin{array}{ll}\mathrm{Cu}^{2+}, & \mathrm{Fe}^{3+}, \\
\text { nitroaromatic } & \\
\text { compounds } & \\
\end{array}$ & $* *$ & 10 \\
\hline$\left[\mathrm{Eu}(\mathrm{pdc})_{1.5}(\mathrm{dmf})\right] \cdot(\mathrm{DMF})_{0.5}\left(\mathrm{H}_{2} \mathrm{O}\right)_{0.5}$ & $\mathrm{Cu}^{2+}$ & pyridyl sites & 11 \\
\hline$\left\{\left[\mathrm{Eu}_{2}(\mathrm{~N}-\mathrm{BDC})_{3}(\mathrm{dmf})_{4}\right]\right\}_{\mathrm{n}} /$ Eu-MOF-LIC-1 & $\mathrm{Cu}^{2+}, \mathrm{Fe}^{3+}, \mathrm{Al}^{3+}$ & reservedN sites & 12 \\
\hline $0.5 \mathrm{Eu}^{3+} @$ Uio-66(Zr)-(COOH $)_{2}$ & $\mathrm{Cd}^{2+}$ & carboxylic groups & 13 \\
\hline $\left.\mathrm{CdCl}\left(\mathrm{L}_{3}\right) \mathrm{Eu}_{0.5} \mathrm{~Tb}_{0.5}\left(\mathrm{H}_{2} \mathrm{O}\right)(\mathrm{DMA})\right]\left(\mathrm{NO}_{3}\right) \cdot 3 \mathrm{DMA}$ & Organic molecule & $* *$ & 14 \\
\hline Eu-TCM & Organic molecule & $* *$ & 15 \\
\hline$\left[\mathrm{Tb}(\mathrm{HTADIP})\left(\mathrm{H}_{2} \mathrm{O}\right)_{3}\right] \cdot 2 \mathrm{H}_{2} \mathrm{O}$ & $\mathrm{Cu}^{2+}$ & $\begin{array}{l}\text { carboxylic } \\
\text { groupstriazole group }\end{array}$ & 16 \\
\hline $\mathrm{EuL}_{4}$ & $\mathrm{Fe}^{3+}$ & $* *$ & 17 \\
\hline$\left[\mathrm{Eu}(\mathrm{PDA})_{3} \mathrm{Fe}_{1.5}\left(\mathrm{H}_{2} \mathrm{O}\right)_{3}\right] \cdot 1.5 \mathrm{H}_{2} \mathrm{O}$ & $\mathrm{Mg}^{2+}$ & $* *$ & 18 \\
\hline$\left[\mathrm{Eu}\left(\mathrm{HL}_{5}\right)\left(\mathrm{H}_{2} \mathrm{O}\right)_{2}\right] \cdot 2 \mathrm{H}_{2} \mathrm{O}$ & $\mathrm{Fe}^{3+}$ & carboxylic groups & 19 \\
\hline$\left[\mathrm{Tb}_{4}\left(\mu_{3}-\mathrm{OH}\right)_{4}(\mathrm{BPDC})_{3}(\mathrm{BPDCA})_{0.5}\left(\mathrm{H}_{2} \mathrm{O}\right)_{6}\right] \mathrm{ClO}_{4} \cdot 5 \mathrm{H}_{2} \mathrm{O}$ & 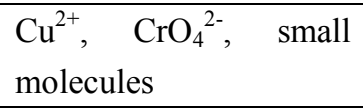 & pyridyl $\mathrm{N}$ atoms & 20 \\
\hline$\left[\mathrm{DyAg}(\mathrm{PDA})_{2}\left(\mathrm{H}_{2} \mathrm{O}\right)_{3}\right] \cdot 3 \mathrm{H}_{2} \mathrm{O}$ & $\mathrm{Mg}^{2+}$, small molechles & $* *$ & 21 \\
\hline
\end{tabular}

$\mathrm{H}_{3} \mathrm{BTPCA}=1,1^{\prime}, 1^{\prime \prime}$-(benzene-1,3,5-triyl)tripiperidine-4-carboxylic acid;

$\mathrm{H}_{2} \mathrm{BDC}=\mathrm{p}$-Benzenedicarboxylic acid;

$\mathrm{H}_{4} \mathrm{BTMIPA}=5,5^{\prime}$-methylenebis(2,4,6-trimethylisophthalic acid);

$\mathrm{Na}_{2} \mathrm{H}_{2} \mathrm{DSOA}=$ Disodium-2,2'-disulfonate-4,4"-oxydibenzoic acid;

$\mathrm{H}_{4}$ btca $=1,10$-biphenyl-2,3,3',5'-tetracarboxylic acid;

$\mathrm{H}_{2} \mathrm{BDC}=$ benzene-1,4-dicarboxylic acid;

$\mathrm{HL}_{2}=4^{\prime}$-(4-carboxyphenyl)-2,2':6',2"-terpyridine;

$\mathrm{H}_{4} \mathrm{BPTA}=\left[1,1^{\prime}\right.$-biphenyl $]-2,2^{\prime}, 5,5^{\prime}$-tetracarboxylic acid;

$\mathrm{Pdc}=$ pyridine-3,5-dicarboxylate;

$\mathrm{H}_{3} \mathrm{~L}_{3}=4,4,4^{\prime \prime}-\left(\left(2,2^{\prime}, 2^{\prime \prime}\right.\right.$-(nitrilotris(methylene))tris(1H-benzo[d]-imidazole-2,1-diyl))tris(methylene))-

tribenzoic acid; 
$\mathrm{H}_{3} \mathrm{TCM}=((2,4,6$-trimethylbenzene-1,3,5-triyl)-tris(methylene) $)$-tris-(oxy))tribenzoic acid;

$\mathrm{H}_{4} \mathrm{~L}_{4}=$ tetrakis[4-(carboxyphenyl)oxamethyl]methane acid;

$\mathrm{H}_{2} \mathrm{PDA}=$ pyridine-2,6-dicarboxylic acid;

$\mathrm{H}_{4} \mathrm{~L}_{5}=3,5$-di-(2,4-dicarboxylphenyl)pyridine;

$\mathrm{BPDC}^{2-}=3,3$-dicarboxylate-2,2-dipyridine anion;

$\mathrm{BPDCA}^{2-}=$ biphenyl-4,4-dicarboxylate anion.

\section{References}

(1) Xu, X.-Y.; Yan. B. ACS Appl. Mater. Interfaces, 2015, 7, 721-729.

(2) Tang, Q.; Liu, S.; Liu, Y.; Miao, J.; Li, S.; Zhang, L.; Shi, Z. Zheng, Z. Inorg. Chem. 2013, 52, 2799-2801.

(3) Wang, R.; Dong, X.-Y.; Xu, H.; Pei, R.-B.; Ma, L.-M.; Zang, S.-Q.; Hou,H.-W.; Mak, T. C. W. Chem. Commun. 2014, 50, 9153-9156.

(4) Wang, Y.; Chu, T.; Yu, M.; Liu, H.; Yang, Y. RSC Adv. 2014, 4, 58178-58183.

(5) Chen, Z.; Sun, Y.; Zhang, L.; Sun, D.; Liu, F.; Meng, Q.; Wang, R.; Sun, D. Chem. Commun. 2013, 49, 11557-11559.

(6) Dong, X.-Y.; Wang, R.; Wang, J.-Z.; Zang, S.-Q.; Mak, T. C. W. J. Mater. Chem. A 2015, 3, $641-647$.

(7) Sun, W.; Wang, J.; Zhang, G.; Liu, Z. RSC Adv. 2014, 4, 55252-55255.

(8) Song, X.-Z.; Song, S.-Y.; Zhao, S.-N.; Hao, Z.-M.; Zhu, M.; Meng, X.; Wu, L.-L.; Zhang, H.-J. Adv. Funct. Mater. 2014, 24, 4034-4041.

(9) Wu, Z.-L.; Dong, J.; Ni, W.-Y.; Zhang, B.-W.; Cui, J.-Z.; Zhao, B. Inorg. Chem., 2015, 54, $5266-5272$.

(10)Wang, W.; Yang, J.; Wang, R.; Zhang, L.; Yu, J.; Sun, D. Cryst. Growth Des. 2015, 15, 2589-2592.

(11)Chen, B.; Wang, L.; Xiao, Y.; Fronczek, F. R.; Xue, M.; Cui, Y.Angew. Chem. Int. Ed. 2009, $48,500-503$.

(12)Hao, J.-N.; Yan, B. J. Mater. Chem. C 2014, 2, 6758-6764.

(13)Hao, J.-N.; Yan, B. Chem. Commun. 2015, 51, 7737-7740.

(14)Zhang, S. R.; Du, D. Y.; Qin, J. S.; Li, S. L.; He, W. W.; Lan, Y. Q.; Su, Z. M. Inorg. Chem. 2014, 53, 8105-8113.

(15)Zhan, C.; Ou, S.; Zou, C.; Zhao, M.; Wu, C. D. Anal. Chem. 2014, 86, 6648-6653. 
(16)Sun, L.; Li, Y.; Liang, Z.; Yu, J.; Xu, R. Dalton Trans. 2012, 41, 12790-12796.

(17)Dang, S.; Ma, E.; Sun, Z.-M.; Zhang, H. J. Mater. Chem. 2012, 22, 16920-16926.

(18)Zhao, B.; Chen, X. Y.; Chen, Z.; Shi, W.; Cheng, P.; Yan, S. P.; Liao, D. Z. Chem. Commun. 2009, 3113-3115.

(19)Liang. Y.-T.; Yang. G.-P.; Liu, B.; Yan, Y.-T.; Xi Z.-P.;Wang, Y.-Y. Dalton Trans. 2015, 44, $13325-13330$.

(20)Hou, J.-M.; Shi, W.; Li, H.-M.; Li, H.; Cheng, P. J. Phys. Chem. C 2014, 118, 416-426.

(21)Zhao, X.-Q.; Zhao, B.; Shi, W.; Cheng, P. CrystEngComm 2009, 11, 1261-1269.

Table S2. Selected bond lengths $(\AA)$ and bond angles (deg) for complex 1-Eu, 1-Tb and 1-Er.

\begin{tabular}{|c|c|c|c|}
\hline \multicolumn{4}{|c|}{ 1-Eu } \\
\hline $\mathrm{Eu}(1)-\mathrm{O}(2) \# 1$ & $2.286(3)$ & $\mathrm{Eu}(1)-\mathrm{O}(3) \# 3$ & $2.548(4)$ \\
\hline $\mathrm{Eu}(1)-\mathrm{O}(2) \# 2$ & $2.286(3)$ & $\mathrm{Eu}(1)-\mathrm{N}(1) \# 4$ & $2.578(4)$ \\
\hline $\mathrm{Eu}(1)-\mathrm{O}(1)$ & $2.291(4)$ & $\mathrm{Eu}(1)-\mathrm{O}(1) \# 3$ & $2.291(4)$ \\
\hline $\mathrm{Eu}(1)-\mathrm{O}(3)$ & $2.548(4)$ & $\mathrm{Eu}(1)-\mathrm{N}(3)$ & $2.985(7)$ \\
\hline $\mathrm{O}(2) \# 1-\mathrm{Eu}(1)-\mathrm{O}(2) \# 2$ & $144.76(19)$ & $\mathrm{O}(1) \# 3-\mathrm{Eu}(1)-\mathrm{N}(1) \# 4$ & $88.95(15)$ \\
\hline $\mathrm{O}(2) \# 1-\mathrm{Eu}(1)-\mathrm{O}(1)$ & $94.20(14)$ & $\mathrm{O}(3)-\mathrm{Eu}(1)-\mathrm{N}(1) \# 4$ & $141.73(14)$ \\
\hline $\mathrm{O}(2) \# 2-\mathrm{Eu}(1)-\mathrm{O}(1)$ & $90.97(14)$ & $\mathrm{O}(3) \# 3-\mathrm{Eu}(1)-\mathrm{N}(1) \# 4$ & $133.77(12)$ \\
\hline $\mathrm{O}(1)-\mathrm{Eu}(1)-\mathrm{O}(1) \# 3$ & $162.9(2)$ & $\mathrm{O}(1)-\mathrm{Eu}(1)-\mathrm{N}(1) \# 4$ & $77.06(14)$ \\
\hline $\mathrm{O}(2) \# 1-\mathrm{Eu}(1)-\mathrm{O}(3)$ & $73.04(13)$ & $\mathrm{O}(2) \# 1-\mathrm{Eu}(1)-\mathrm{N}(1) \# 4$ & $142.58(13)$ \\
\hline $\mathrm{O}(2) \# 2-\mathrm{Eu}(1)-\mathrm{O}(3)$ & $75.06(13)$ & $\mathrm{N}(1) \# 4-\mathrm{Eu}(1)-\mathrm{N}(1) \# 5$ & $71.11(19)$ \\
\hline $\mathrm{O}(1)-\mathrm{Eu}(1)-\mathrm{O}(3)$ & $123.37(13)$ & $\mathrm{O}(2) \# 1-\mathrm{Eu}(1)-\mathrm{N}(3)$ & $72.38(10)$ \\
\hline $\mathrm{O}(1) \# 3-\mathrm{Eu}(1)-\mathrm{O}(3)$ & $73.74(13)$ & $\mathrm{O}(1)-\mathrm{Eu}(1)-\mathrm{N}(3)$ & $98.56(10)$ \\
\hline $\mathrm{N}(1) \# 4-\mathrm{Eu}(1)-\mathrm{N}(3)$ & $144.45(9)$ & $\mathrm{O}(2) \# 2-\mathrm{Eu}(1)-\mathrm{N}(1) \# 4$ & $72.44(14)$ \\
\hline \multicolumn{4}{|c|}{$1-\mathrm{Tb}$} \\
\hline $\mathrm{Tb}(1)-\mathrm{O}(2) \# 1$ & $2.275(3)$ & $\mathrm{Tb}(1)-\mathrm{O}(3)$ & $2.540(4)$ \\
\hline $\mathrm{Tb}(1)-\mathrm{O}(2) \# 2$ & $2.275(3)$ & $\mathrm{Tb}(1)-\mathrm{O}(3) \# 3$ & $2.540(4)$ \\
\hline $\mathrm{Tb}(1)-\mathrm{O}(1) \# 3$ & $2.279(3)$ & $\mathrm{Tb}(1)-\mathrm{N}(1) \# 4$ & $2.565(4)$ \\
\hline $\mathrm{Tb}(1)-\mathrm{O}(1)$ & $2.279(3)$ & $\mathrm{Tb}(1)-\mathrm{N}(1) \# 5$ & $2.565(4)$ \\
\hline $\mathrm{O}(2) \# 1-\mathrm{Tb}(1)-\mathrm{O}(2) \# 2$ & $144.90(18)$ & $\mathrm{N}(1) \# 4-\mathrm{Tb}(1)-\mathrm{N}(1) \# 5$ & $71.14(17)$ \\
\hline $\mathrm{O}(2) \# 1-\mathrm{Tb}(1)-\mathrm{O}(1)$ & $93.86(12)$ & $\mathrm{O}(2) \# 1-\mathrm{Tb}(1)-\mathrm{N}(1) \# 4$ & $142.41(13)$ \\
\hline $\mathrm{O}(2) \# 2-\mathrm{Tb}(1)-\mathrm{O}(1)$ & $91.22(12)$ & $\mathrm{O}(2) \# 2-\mathrm{Tb}(1)-\mathrm{N}(1) \# 4$ & $72.43(12)$ \\
\hline $\mathrm{O}(1) \# 3-\mathrm{Tb}(1)-\mathrm{O}(1)$ & $163.09(19)$ & $\mathrm{O}(1) \# 3-\mathrm{Tb}(1)-\mathrm{N}(1) \# 4$ & $89.32(14)$ \\
\hline $\mathrm{O}(2) \# 1-\mathrm{Tb}(1)-\mathrm{O}(3)$ & $72.82(12)$ & $\mathrm{O}(1)-\mathrm{Tb}(1)-\mathrm{N}(1) \# 4$ & $76.86(13)$ \\
\hline $\mathrm{O}(2) \# 2-\mathrm{Tb}(1)-\mathrm{O}(3)$ & $75.48(12)$ & $\mathrm{O}(3)-\mathrm{Tb}(1)-\mathrm{N}(1) \# 4$ & $142.22(12)$ \\
\hline $\mathrm{O}(1) \# 3-\mathrm{Tb}(1)-\mathrm{O}(3)$ & $73.40(12)$ & $\mathrm{O}(3) \# 3-\mathrm{Tb}(1)-\mathrm{N}(1) \# 4$ & $133.09(12)$ \\
\hline $\mathrm{O}(1)-\mathrm{Tb}(1)-\mathrm{O}(3)$ & $123.51(12)$ & & \\
\hline \multicolumn{4}{|c|}{ 1-Er } \\
\hline $\operatorname{Er}(1)-\mathrm{O}(2) \# 1$ & $2.233(3)$ & $\operatorname{Er}(1)-\mathrm{O}(3) \# 3$ & $2.497(3)$ \\
\hline $\operatorname{Er}(1)-\mathrm{O}(2) \# 2$ & $2.233(3)$ & $\operatorname{Er}(1)-\mathrm{N}(1) \# 4$ & $2.517(4)$ \\
\hline
\end{tabular}




\begin{tabular}{|l|l|l|l|}
\hline $\mathrm{Er}(1)-\mathrm{O}(1) \# 3$ & $2.234(3)$ & $\operatorname{Er}(1)-\mathrm{O}(3)$ & $2.497(3)$ \\
\hline $\mathrm{Er}(1)-\mathrm{O}(1)$ & $2.234(3)$ & $\operatorname{Er}(1)-\mathrm{N}(3)$ & $2.922(7)$ \\
\hline $\mathrm{O}(2) \# 1-\operatorname{Er}(1)-\mathrm{O}(2) \# 2$ & $144.90(17)$ & $\mathrm{O}(1)-\operatorname{Er}(1)-\mathrm{N}(1) \# 4$ & $76.77(13)$ \\
\hline $\mathrm{O}(2) \# 1-\operatorname{Er}(1)-\mathrm{O}(1)$ & $93.37(11)$ & $\mathrm{O}(3)-\operatorname{Er}(1)-\mathrm{N}(1) \# 4$ & $142.23(11)$ \\
\hline $\mathrm{O}(2) \# 2-\operatorname{Er}(1)-\mathrm{O}(1)$ & $91.84(12)$ & $\mathrm{O}(3) \# 3-\operatorname{Er}(1)-\mathrm{N}(1) \# 4$ & $132.98(11)$ \\
\hline $\mathrm{O}(1) \# 3-\operatorname{Er}(1)-\mathrm{O}(1)$ & $162.67(17)$ & $\mathrm{O}(1) \# 3-\operatorname{Er}(1)-\mathrm{N}(1) \# 4$ & $89.05(13)$ \\
\hline $\mathrm{O}(2) \# 1-\operatorname{Er}(1)-\mathrm{O}(3)$ & $72.99(11)$ & $\mathrm{O}(1) \# 3-\operatorname{Er}(1)-\mathrm{N}(1) \# 5$ & $76.77(12)$ \\
\hline $\mathrm{O}(2) \# 2-\operatorname{Er}(1)-\mathrm{O}(3)$ & $75.37(11)$ & $\mathrm{N}(1) \# 4-\operatorname{Er}(1)-\mathrm{N}(1) \# 5$ & $70.97(16)$ \\
\hline $\mathrm{O}(1) \# 3-\operatorname{Er}(1)-\mathrm{O}(3)$ & $73.37(12)$ & $\mathrm{O}(2) \# 1-\operatorname{Er}(1)-\mathrm{N}(3)$ & $72.45(9)$ \\
\hline $\mathrm{O}(1)-\operatorname{Er}(1)-\mathrm{O}(3)$ & $123.96(11)$ & $\mathrm{O}(1)-\operatorname{Er}(1)-\mathrm{N}(3)$ & $98.67(9)$ \\
\hline $\mathrm{O}(2) \# 1-\operatorname{Er}(1)-\mathrm{N}(1) \# 4$ & $142.26(12)$ & $\mathrm{N}(1) \# 4-\operatorname{Er}(1)-\mathrm{N}(3)$ & $144.52(8)$ \\
\hline $\mathrm{O}(2) \# 2-\operatorname{Er}(1)-\mathrm{N}(1) \# 4$ & $72.56(12)$ & & \\
\hline $\mathrm{Sy}$ & & & \\
\hline
\end{tabular}

Symmetry codes for 1-Eu:\#1 -x,-y,-z; \#2 x,-y,z+1/2; \#3 -x,y,-z+1/2; \#4 -x+1/2,-y+1/2,-z+1; \#5 $\mathrm{x}-1 / 2,-\mathrm{y}+1 / 2, \mathrm{z}-1 / 2$. For 1-Tb: \#1 -x+1,-y+1,-z+1; \#2 $\mathrm{x},-\mathrm{y}+1, \mathrm{z}-1 / 2 ; \quad \# 3-\mathrm{x}+1, \mathrm{y},-\mathrm{z}+1 / 2 ; \quad \# 4$ $-\mathrm{x}+1 / 2,-\mathrm{y}+1 / 2,-\mathrm{z} ; \# 5 \mathrm{x}+1 / 2,-\mathrm{y}+1 / 2, \mathrm{z}+1 / 2$. For 1-Er: \#1 -x,-y,-z+1; \#2 x,-y,z+1/2; \#3 -x,y,-z+3/2; $\# 4-\mathrm{x}+1 / 2,-\mathrm{y}+1 / 2,-\mathrm{z}+2 ; \quad \# 5 \mathrm{x}-1 / 2,-\mathrm{y}+1 / 2, \mathrm{z}-1 / 2$.

Table S3. Comparison of the detective sensitivity and responsive time in various $\mathrm{Fe}^{3+}$ sensors.

\begin{tabular}{llll}
\hline Molecular & Sensitivity & Responsive time & Reference \\
\hline MIL-53(Al) & $0.9 \times 10^{-6} \mathrm{M}$ & Seconds & 1 \\
{$[\mathrm{~Tb}(\mathrm{BTB})(\mathrm{DMF})]$} & $1 \times 10^{-5} \mathrm{M}$ & Seconds & 2 \\
{$\left[\mathrm{Eu}(\mathrm{BTPCA})\left(\mathrm{H}_{2} \mathrm{O}\right)\right]$} & $1 \times 10^{-5} \mathrm{M}$ & Seconds & 3 \\
{$\left[\mathrm{~Tb}(\mathrm{Hbtca})\left(\mathrm{H}_{2} \mathrm{O}\right)_{2}\right]$} & $1 \times 10^{-5} \mathrm{M}$ & $5 \mathrm{~min}$ & 4 \\
{$\left[\mathrm{Eu}\left(\mathrm{H}_{2} \mathrm{O}\right)_{2}(\mathrm{BTMIPA})\right]$} & $1 \times 10^{-5} \mathrm{M}$ & $* *$ & 5 \\
Tb-DSOA & $1 \times 10^{-5} \mathrm{M}$ & $* *$ & 6 \\
This work & $2.6 \times 10^{-5} \mathrm{M}$ & Seconds & \\
Eu(C $\left.\mathrm{C}_{22} \mathrm{H}_{14} \mathrm{~N}_{3} \mathrm{O}_{2}\right)_{3}$ & $1 \times 10^{-4} \mathrm{M}$ & $20 \mathrm{~h}$ & 7 \\
{$\left[\left(\mathrm{H}_{2} \mathrm{NMe}\right)\right]\left[\mathrm{Eu}\left(\mathrm{C}_{33} \mathrm{H}_{24} \mathrm{O}_{12}\right)\left(\mathrm{H}_{2} \mathrm{O}\right)\right]$} & $2 \times 10^{-4} \mathrm{M}$ & $8 \mathrm{~h}$ & 8 \\
$\mathrm{Fe}(\mathrm{acac})_{3} \subset \mathrm{Zn}\left(\mathrm{C}_{15} \mathrm{H}_{12} \mathrm{NO}_{2}\right)_{2}$ & $5 \times 10^{-3} \mathrm{M}$ & $0.5 \mathrm{~h}$ & 9 \\
\hline
\end{tabular}

$\mathrm{H}_{3} \mathrm{BTB}=$ benzene-1,3,5-tribenzoate;

$\mathrm{H}_{3} \mathrm{BTPCA}=1,1,1$-(benzene-1,3,5-triyl)tripiperidine-4-carboxylic acid;

$\mathrm{H}_{4}$ btca $=1,1^{\prime}$-biphenyl-2,3,3',5'-tetracarboxylic acid;

$\mathrm{H}_{4} \mathrm{BTMIPA}=5,5^{\prime}$-methylenebis(2,4,6-trimethylisophthalic acid;

$\mathrm{H}_{4} \mathrm{DSOA}=$ sulfonate-carboxylateacid.

\section{References}

(1) Yang, C. X.; Ren, H. B.; Yan, X. P. Anal. Chem. 2013,85, 7441-7446.

(2) Xu, H.; Hu, H. C.; Cao, C. S.; Zhao, B. Inorg. Chem. 2015,54, 4585-4587.

(3) Tang, Q.; Liu, S.; Liu, Y.; Miao, J.; Li, S.; Zhang, L.; Shi, Z.; Zheng, Z. Inorg. Chem. 2013, 52, 2799-2801.

(4) Sun, W.; Wang, J.; Zhang, G.; Liu, Z. RSC Adv., 2014, 4, 55252-55255. 
(5) Chen, Z.; Sun, Y.; Zhang, L.; Sun, D.; Liu, F.; Meng, Q.; Wang, R.; Sun, D. Chem. Commun. 2013, 49, 11557-11559.

(6) Dong, X.-Y.; Wang, R.; Wang, J.-Z.; Zang, S.-Q.; Mak, T. C. W. J. Mater. Chem. A, 2015, 3, 641-647.

(7) Zheng, M.; Tan, H.; Xie, Z.; Zhang, L.; Jing, X.; Sun, Z. ACS Appl. Mater. Interfaces, 2013, 5, 1078-1083.

(8) Dang, S.; Ma, E.; Sun, Z.-M.; Zhang, H. J. Mater.Chem., 2012, 22, 16920-16926.

(9) Hou, G. G.; Liu, Y.; Liu, Q. K.; Ma, J. P.; Dong, Y. B. Chem. Commun. 2011, 47, 10731-10733.

Table S4. Comparison of the isosteric heat $\left(\mathrm{Q}_{\mathrm{st}}\right)$ of 1-Eu with the selected MOFs.

\begin{tabular}{|c|c|c|}
\hline Complexes & $Q_{\mathrm{st}} / \mathrm{kJ} \mathrm{mol}^{-1}$ & Ref \\
\hline $\mathrm{Zn}_{2}\left(\mathrm{C}_{2} \mathrm{O}_{4}\right)\left(\mathrm{C}_{2} \mathrm{~N}_{4} \mathrm{H}_{3}\right)_{2}\left(\mathrm{H}_{2} \mathrm{O}\right)_{0.5}{ }^{\mathrm{c}}$ & 71 & 1 \\
\hline UiO-66-EA ${ }^{\mathrm{c}}$ & 66 & 2 \\
\hline$\left[\mathrm{Co}_{2}(\mathrm{DHTP})\right]^{\mathrm{a}}$ & 50.1 & 3 \\
\hline $\mathrm{Mg} / \mathrm{DOBDC}^{\mathrm{a}}$ & 47 & 4 \\
\hline$\left[\mathrm{Cu}_{2}(\mathrm{~m}-\mathrm{OH})_{2}(\mathrm{bdim})\right] \cdot 5.4 \mathrm{H}_{2} \mathrm{O}$ & 46.7 & 5 \\
\hline MPM-1-TIFSIX & 44.4 & 6 \\
\hline $\operatorname{Mg}_{2}($ dobpdc $)$ & 44 & 1 \\
\hline $\mathrm{Mg}_{2}(\mathrm{dobdc})^{\mathrm{a}}$ & 42 & 7 \\
\hline $\mathrm{HOF}-3^{\mathrm{a}}$ & 42 & 8 \\
\hline $\mathrm{Zn}_{2}\left(\mathrm{C}_{2} \mathrm{O}_{4}\right)\left(\mathrm{C}_{2} \mathrm{~N}_{4} \mathrm{H}_{3}\right)_{2}\left(\mathrm{H}_{2} \mathrm{O}\right)_{0.5}{ }^{\mathrm{c}}$ & 40.8 & 9 \\
\hline This work ${ }^{\mathrm{d}}$ & 39.2 & \\
\hline$\left[\mathrm{Ni}(\mathrm{dcpy})(\mathrm{bpe}) 0.5\left(\mathrm{H}_{2} \mathrm{O}\right)\right] \cdot 3 \mathrm{H}_{2} \mathrm{O}^{\mathrm{a}, \mathrm{d}, \mathrm{e}}$ & 34.6 & 10 \\
\hline$\left\{\left[\mathrm{Cu}_{3}(\mathrm{CPT})_{4}\left(\mu_{3}-\mathrm{OH}\right)\right] \cdot \mathrm{NO}_{3} \cdot 7 \mathrm{H}_{2} \mathrm{O} \cdot \mathrm{EtOH}\right\}_{\mathrm{n}}$ & 32 & 11 \\
\hline HKUST- $1^{\text {a }}$ & 30.4 & 3 \\
\hline $\mathrm{TbL}^{\mathrm{b}}$ & 28.23 & 12 \\
\hline SNU- $151^{\mathrm{c}}$ & 27.1 & 13 \\
\hline
\end{tabular}

a, open metal sites; b, imidazolyl active sites; c, amine groups; d, oxygen sites; e, pyridine groups.

DHTP $=2,5$-dihydroxyterephthalate;

DOBDC $=2,5$-dioxido-1,4-benzene-dicarboxylate;

$\mathrm{H}_{2}$ bdim = benzodiimi-dazole;

dobpdc $^{4-}=4,4^{\prime}$-dioxido-3,3'-biphenyldicarboxylate;

$\operatorname{dobdc}^{4-}=1,4$-dioxido-2,5-benzenedicarboxylate;

$\mathrm{H}_{2} \mathrm{dcpy}=3$-(2,5-dicarboxylphenyl)pyridine acid,

bpe =1,2-bi(4-pyridyl)ethane;

HCPT = 4-(4-carboxyphen-yl)-1,2,4-triazole;

$\mathrm{H}_{3} \mathrm{~L}=3$-(3,5-dicarboxylphenyl)-5-(4-carboxylphenyl)-1-H-1,2,4-triazole.

\section{References}


(1) McDonald, T. M.; Lee, W. R.; Mason, J. A.; Wiers, B. M.; Hong, C. S.; Long, J. R. J. Am. Chem. Soc. 2012, 134, 7056-7065.

(2) Li, L.-J.; Liao, P.-Q.; He, C.-T.; Wei, Y.-S.; Zhou, H.-L.; Lin, J.-M.; Li, X.-Y.; Zhang, J.-P. J. Mater. Chem. A2015, 3, 21849-21855.

(3) Xiang, S.; Zhou, W.; Zhang, Z.; Green, M. A.; Liu, Y.; Chen, B. Angew. Chem. Int. Ed. 2010, $49,4615-4618$.

(4) Caskey, S. R.; Wong-Foy, A. G.; Matzger, A. J. J. Am. Chem. Soc. 2008, 130, 10870-10871.

(5) Zhou, D.-D.; He, C.-T.; Liao, P.-Q.; Xue, W.; Zhang, W.-X.; Zhou, H.-L.; Zhang, J.-P.; Chen, X.-M. Chem. Commun., 2013, 49, 11728-11730.

(6) Nugent, P. S.; Rhodus, V. L.; Pham, T.; Forrest, K.; Wojtas, L.; Space, B.; Zaworotko, M. J. J. Am. Chem. Soc. 2013, 135, 10950-10953.

(7) Mason, J. A.; Sumida, K.; Herm, Z. R.; Krishna, R.; Long, J. R. Energy Environ. Sci., 2011, 4, 3030-3040.

(8) Li, P.; He, Y.; Zhao, Y.; Weng, L.; Wang, H.; Krishna, R.; Wu, H.; Zhou, W.; O'Keeffe, M.; Han, Y.; Chen, B. Angew. Chem. Int. Ed. 2015, 54, 574-577.

(9) Vaidhyanathan, R.; Iremonger, S. S.; Dawson, K. W.; Shimizu, G. K. Chem. Commun. 2009, $5230-5232$

(10)Du, L. Y.; Wang, H.; Liu, G.; Xie, D.; Guo, F. S.; Hou, L.; Wang, Y. Y. Cryst. Growth Des., 2014, 14, 1110-1127.

(11)Chen, D. M.; Zhang, X. P.; Shi, W.; Cheng, P. Inorg. Chem., 2015, 54, 5512-5518.

(12)Yang, Y.; Jiang, F.; Chen, L.; Pang, J.; Wu, M.; Wan, X.; Pan, J.; Qian, J.; Hong, M. J. Mater. Chem. A, 2015,3, 13526-13532.

(13)Choi, M. H.; Park, H. J.; Hong, D. H.; Suh, M. P. Chem. Eur. J. 2013, 19, 17432-17438.

Table S5. The atomic partial charges (e) in 1-Eu.

\begin{tabular}{|l|l|l|l|l|l|}
\hline Eu1 & 1.15847 & C2 & -0.0357080 & H2 & 0.315690 \\
\hline N1 & -0.106214 & C3 & -0.165315 & H3 & 0.131984 \\
\hline N2 & -0.417497 & C4 & -0.00469190 & H5 & 0.187658 \\
\hline N3 & 0.282505 & C5 & -0.113769 & H6 & 0.134632 \\
\hline O1 & -0.435626 & C6 & -0.171901 & H7 & 0.152020 \\
\hline O2 & -0.397066 & C7 & -0.149188 & H9 & 0.0996128 \\
\hline O3 & -0.296144 & C8 & 0.0933672 & H10 & 0.122517 \\
\hline O4 & -0.459292 & C9 & -0.155536 & & \\
\hline C1 & 0.644300 & C10 & 0.0760366 & & \\
\hline
\end{tabular}

